Pacific Journal of Mathematics

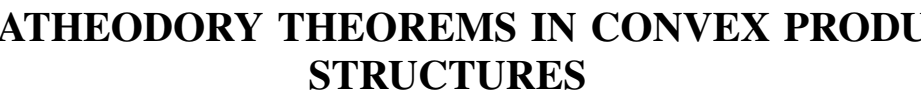




\title{
CARATHEODORY THEOREMS IN CONVEX PRODUCT STRUCTURES
}

\author{
JOHN R. REAY
}

\begin{abstract}
Various attempts have been made to place convexity in an axiomatic setting. Recently J. Eckhoff has considered the classic theorem of Radon in several different settings. Most of his work is done in what we call an Eckhoff space, i.e., in a finite product of euclidean spaces where convex sets are defined as the cartesian products of usual convex sets in each component space. The purpose of this paper is to investigate the closely related theorem of Caratheodory and its generalizations in this setting.
\end{abstract}

The papers of F.W. Levi [5] and Dauzer, Grunbaum, Klee [3] have various approaches to axiomatic settings of convexity, and a good bibliography for before 1961. See the papers of Eckhoff [4] and Bonnice-Reay [2] for more recent results and references.

1. Eckhoff spaces. The pair $(E, \mathscr{C})$ denotes an Eckhoff space provided (1) $E$ is a direct cartesian product $E=\prod_{i=1}^{n} E_{i}$ where each $E_{i}$ is a $d_{i}$-dimensional euclidean space with $\mathscr{C}_{i}$ the family of all convex sets of $E_{i}$, and (2) $\mathscr{C}=\left\{\prod_{i=1}^{n} A_{i}: A_{i} \in \mathscr{C}_{i}\right\}$ is the family of all productconvex sets in $E$. For any set $X \subset E$, the set $E(X)=\cap\{A: X \subset A \in \mathscr{E}\}$ is called the product-convex hull of $X$. Let $\pi_{i}: E \rightarrow E_{i}$ denote the usual projection. Then we can consider $E$ as a linear space of dimension $d=\sum_{i=1}^{n} d_{i}$, and $E(X)=\prod_{i=1}^{n}\left(\operatorname{conv} \pi_{i} X\right)$ where conv $B$ denotes the usual convex hull of $B$ in each euclidean space $E_{i}$. The cardinality of $B$ will be denoted by $|B|$. Using the notation of Bonnice-Klee [1] and others, we say that $\operatorname{int}_{r} B$ is the set of all points $p$ for which there exists an $r$-dimensional simplex contained in $B$ and containing $p$ in its relative interior.

2. Caratheodory-type theorems. By a Caratheodory-type theorem we mean a result which asserts that if a point is embedded in the (axiomatically defined) hull of a set $X$, then it is similarly embedded in the hull of a sufficiently small subset of $X$. Note that the case $n=1$ of Theorem 1 below is the result usually called Caratheodory's theorem.

THEOREM 1. If $X$ is any subset of an Eckhoff space $E=\prod_{i=1}^{n} E_{i}$ of dimension $d=\Sigma d_{i}$ and if $p \in E(X)$, then $p \in E(Y)$ for some $Y \subset X$ with $|Y| \leqq d+\delta$, where $\delta=1$ if $n=1$ and $\delta=0$ if $n>1$. Fur- 
thermore, if $m=|Y|$ is the cardinality of a smallest subset $Y$ of $X$ for which $p \in E(Y)$, then $p \in \operatorname{int}_{r} E(Y)$ where

$$
\max (0, m-n) \leqq r \leqq(m-1)\left|\left\{d_{i}: d_{i} \geqq m\right\}\right|+\Sigma_{d_{i}<m} d_{i} .
$$

Proof. If $n=1$ the upper and lower bounds on $r$ reduce to $r=$ $m-1$, that is, $p$ lies interior to the $(m-1)$-simplex determined by the $m$ points of $Y$.

Assume $n=2$. It suffices to show that there is a set $Y \subset X$ for which $|Y| \leqq d_{1}+d_{2}=d$ and $\pi_{i} p \in \operatorname{conv} \pi_{i} Y$ for $i=1$, 2. ApplyingCaratheodory's theorem to $E_{1}$, there is a subset $Y_{1} \subset X$ with $\left|Y_{1}\right| \leqq$ $d_{1}+1$ for which $\pi_{1} p \in \operatorname{conv} \pi_{1} Y_{1}$. Now if $\pi_{2} p \in \operatorname{conv} \pi_{2} Y_{1}$ as well, then $p \in E\left(Y_{1}\right)$ and we are done. Otherwise choose a set $Y_{2} \subset X$ of minimal cardinality such that $\pi_{2} p \in \operatorname{conv} \pi_{2}\left(Y_{1} \cup Y_{2}\right)$. Since we may choose one of the $d_{2}+1$ points of Caratheodory's theorem arbitrarily (see [6], Lemmas 4.1-4.4) it follows that $\left|Y_{2}\right| \leqq d_{2}$. Thus letting $Y=Y_{1} \cup Y_{2}$ it follows that $p \in E(Y)$ and $|Y| \leqq\left|Y_{1}\right|+\left|Y_{2}\right| \leqq d_{1}+d_{2}+1$. We are therefore done unless both $\left|Y_{1}\right|=d_{1}+1$ and $\left|Y_{2}\right|=d_{2}$ and $Y_{1} \cap Y_{2}=\varnothing$. In this case we will show that one correctly chosen point may be removed from $Y_{1} \cup Y_{2}$.

Case 1. $\pi_{2} p \in \operatorname{conv} \pi_{2} Y_{2}$. In this case we reverse the roles of $E_{1}$ and $E_{2}$ in the above argument, i.e., let $Y_{2}$ be as above and redefine $Y_{1}$ to be a set of minimal cardinality so that $\pi_{1} p \in \operatorname{conv}\left(Y_{2} \cup Y_{1}\right)$. Then $\left|Y_{1}\right| \leqq d_{1}$ and $\left|Y_{2}\right|=d_{2}$ and $p \in E\left(Y_{2} \cup Y_{1}\right)$.

Case 2. $\pi_{2} p \notin \operatorname{conv} \pi_{2} Y_{2}$. In this case $\left|Y_{2}\right|=d_{2}$ so for each point $y \in Y_{1}$ it is true that $\pi_{2} p \in \operatorname{conv} \pi_{2}\left(\{y\} \cup Y_{2}\right)$. Thus some point of $\pi_{1} Y_{2}$ in the space $E_{1}$ may be used to replace a particular point of $Y_{1}$, say $y_{1}$. Then $\pi_{1} p \in \operatorname{conv} \pi_{1}\left(Y_{2} \cup\left(Y_{1}-\left\{y_{1}\right\}\right)\right)$ and it is still true that $\pi_{2} p \in$ conv $\pi_{2}\left(\left(Y_{1}-\left\{y_{1}\right\}\right) \cup Y_{2}\right)$. This establishes Case 2, and hence proves the first statement of the theorem if $n=2$.

The case when $n \geqq 3$ now follows easily. As in the case $n=2$ there exists a set $Y_{1} \cup Y_{2} \subset X$ for which $\pi_{i} p \in \operatorname{conv} \pi_{i}\left(Y_{1} \cup Y_{2}\right)$ for $i=1,2$ and $\left|Y_{1} \cup Y_{2}\right| \leqq d_{1}+d_{2}$. For each $i \geqq 3$ there is a set $Y_{i} \subset X$, by Caratheodory's theorem in $E_{i}$, such that $\left|Y_{i}\right| \leqq d_{i}$ and $\pi_{i} p \in \operatorname{conv} \pi_{i}\left(Y_{1} \cup Y_{2} \cup Y_{i}\right)$. The set $Y=\bigcup_{i=1}^{n} Y_{i}$ then has the desired properties; $p \in E(Y)$ and $|Y| \leqq \sum_{i=1}^{n} d_{i}=d$. This establishes the first half of the theorem.

To prove the last statement, let $Y \subset X$ be a smallest subset for which $p \in E(Y)$ and suppose $|Y|=m$. Then for each $i, \pi_{i} p \in \operatorname{int}_{r(i)}$ conv $\pi_{i} Y$ for some largest nonnegative integer $r(i) \leqq d_{i}$, and $p \in \operatorname{int}_{r} E(Y)$ where $r=\sum_{i=1}^{n} r(i)$. It follows that $r$ assumes a minimal value whenever each $r(i)$ is as small as possible, within the constraint $|Y|=m$. 
This is achieved when the points of $Y$ are used as "inefficiently as possible", specifically, when for some partition $Y=Y_{1} \cup \cdots \cup Y_{n}$ we have $\pi_{i} p \in \operatorname{conv} \pi_{i} Y_{i}$ and the points of $\pi_{i}\left(Y \sim Y_{i}\right)$ are not used in $E_{i}$. For example, if $\pi_{i}\left(Y \sim Y_{i}\right)$ is a single point in $E_{i}$ then the points of $\pi_{i} Y$ are the vertices of a simplex in $E_{i}$ of dimension $\left|Y_{i}\right|$, and $\pi_{i} p$ is interior to the subsimplex conv $\pi_{i} Y_{i}$. In any case, Caratheodory's theorem (case $n=1$ ) implies that $r(i)=\left|Y_{i}\right|-1$. Thus $r=\Sigma_{i} r(i)=$ $\left(\Sigma_{i}\left|Y_{i}\right|\right)-n=m-n$, and in general $r \geqq m-n$. The other inequality on $r$ follows from the fact that the $m$ points of $Y$ projected onto each space $E_{i}$ can have a convex hull of dimension at most $\min \left(m-1, d_{i}\right)$ in $E_{i}$. This proves Theorem 1 .

ExAmples. The following examples show that the bounds in Theorem 1 cannot, in general, be improved.

(1) For each $i=1,2, \cdots, n$, let $X_{i}$ be a subset of $E_{i}$ for which $X_{i} \cup\{0\}$ form the vertices of a nondegenerate $d_{i}$-simplex, and let $p_{i}$ be in the relative interior of the simplex conv $X_{i}$. Define $p \in E$ by the relations $\pi_{i} p=p_{i}$. For each point $x_{i}$ in each set $X_{i}$ define the point $\bar{x}_{i} \in E$ by the relations $\pi_{i} \bar{x}_{i}=x_{i}$ and $\pi_{j} \bar{x}_{i}=0$ if $j \neq i$. Let $X \subset E$ be the set of all such points $\bar{x}_{i}$. Then clearly $p \in E(X)$, but $p \notin E(Y)$ for any proper subset of $Y$ of $X$, and $|X|=\Sigma\left|X_{i}\right|=\Sigma d_{i}=d$. Furthermore $p \in \operatorname{int}_{r} E(X)$ where

$$
r=\Sigma\left(\left|X_{i}\right|-1\right)=|X|-n=m-n .
$$

(2) As a second example, let $m$ be any integer for which $1 \leqq$ $m \leqq \max \left\{d_{i}+1: i=1, \cdots, n\right\}$. For each subspace $E_{i}$ if $m \leqq d_{i}+1$ let $\left\{x_{i j}\right\}_{j=1}^{m}$ be the vertices of a nondegenerate simplex in $E_{i}$. If $m>d_{i}+1$ let $\left\{x_{i j}: j=1, \cdots, d_{i}+1\right\}$ be the vertices of a nondegenerate simplex, and let $x_{i 1}=x_{i j}$ for $j=\left(d_{i}+2\right), \cdots, m$. In either case choose a point $p_{i}$ in the relative interior of this simplex. Now define $p \in E$ by the relations $\pi_{i} p=p_{i}$ and let $X=\left\{x_{j}\right\}_{j=1}^{m} \subset E$ where each $x_{j}$ is defined by $\pi_{i} x_{j}=x_{i j} \in E_{i}$. Then $|X|=m$ and $p \in E(X)$ but $p \notin E(Y)$ for any proper subset $Y$ of $X$. Also $p \in$ int $_{r} E(X)$ where

$$
r=(m-1)\left|\left\{d_{i}: d_{i} \geqq m\right\}\right|+\Sigma_{d_{i}<m} d_{i} .
$$

The case where $n=1$ and $r=d$ in Theorem 2 below is commonly called Steinitz's theorem.

THEOREM 2. If $X$ is any subset of an Eckhoff space $E=\prod_{i=1}^{n} E_{i}$ and if $r \geqq 0$ is the largest integer for which $p \in \operatorname{int}_{r} E(X)$, then $p \in \operatorname{int}_{r} E(Y)$ for some subset $Y \subset X$ with $|Y| \leqq 2 r+\delta$ where $\delta$ is the number of spaces $E_{i}$ for which $\pi_{i} p \notin$ int $_{1} \operatorname{conv} \pi_{i} X$. 
Proof. Let $r(i)$ be the largest integer for which $\pi_{i} p \in \operatorname{int}_{r(i)} \operatorname{conv} \pi_{i} X$. Thus $r=\Sigma r(i)$. By the Bonnice-Klee theorem (see [1], Th. 2.5) and the maximality of $r(i)$ there is a subset $Y_{i}$ of $X$ for which $\pi_{i} p \in$ int $_{r(i)}$ conv $\pi_{i} Y_{i}$ and $\left|Y_{i}\right| \leqq 2 r(i)$ if $r(i)>0$, and $\left|Y_{i}\right|=1$ if $r(i)=0$. Thus letting $Y=\bigcup_{i=1}^{n} Y_{i}$ we have $p \in \operatorname{int}_{r} E(Y)$ and $|Y|=\left|\cup Y_{i}\right| \leqq \Sigma\left|Y_{i}\right| \leqq$ $2 \Sigma_{r(i)>0} r(i)+\Sigma_{\{i \mid r(i)=0\}} 1=2 r+\delta$. This proves Theorem 2.

Using the techniques from the examples given above, it is easy to construct sets $X$ in Eckhoff spaces which show that the bounds of Theorem 2 cannot, in general, be improved. A further generalization may be obtained by considering $p \in$ int $_{s} E(X)$ where $0<s<r$, and ask the cardinality of the smallest $Y \subset X$ for which $p \in \operatorname{int}_{s} E(Y)$. This is the spirit of the Bonnice-Klee Theorem (see [1] and [6]). Another approach is to add further information about the set $X$, and ask how the bound on $|Y|$ may be improved. For example, if it is known that $k_{i}$ is the dimension of the highest-dimensional simplex with vertices in $\pi_{i} X \subset E_{i}$ and having $\pi_{i} p$ in its relative interior, then the bound on $|Y|$ can, in general, be improved. See Bonnice-Reay [2] for a bibliography and results of this type. Also connectedness or symmetry conditions on $X$ may lead to an improvement of the bound on $|Y|$. See [6] for a bibliography and results of this type.

These theorems and others which depend even more upon the structure of $X$ are similar to the above theorems, but are much more complicated and are therefore omitted.

\section{REFERENCES}

1. W. Bonnice and V. Klee, The generation of convex hulls, Math. Ann., 152 (1963), $1-29$.

2. W. Bonnice and J. Reay, Relative interiors of convex hulls, Proc. Amer. Math. Soc., 20 (1969), 246-250.

3. L. Danzer, B. Grunbaum, and V. Klee, Helly's theorem and its relatives, Proc. of Symp. in Pure Math., vol. 7, "Convexity”, Amer. Math. Soc., 1963, 101-180.

4. J. Eckhoff, Der Satz von Radon in konvexen Productstrukturen I-II, Monatsh. Math., 72 (1968), 303-314; 73 (1969), 7-30.

5. F. W. Levi, On Helly's theorem and the axioms of convexity, J. Indian Math. Soc., (N.S.) 15 (1951), 65-76.

6. J.R. Reay, Generalizations of a theorem of Caratheodory, Amer. Math. Soc. Memoir No. 54, 1965.

Received October 16, 1969.

Western Washington State College 


\section{PACIFIC JOURNAL OF MATHEMATICS}

\section{EDITORS}

H. SAmelson

Stanford University

Stanford, California 94305

RICHARd PieRCe

University of Washington

Seattle, Washington 98105

\author{
J. DugundJI \\ Department of Mathematics \\ University of Southern California \\ Los Angeles, California 90007 \\ RICHARD ARENS \\ University of California \\ Los Angeles, California 90024
}

ASSOCIATE EDITORS
E. F. BECKENBACH
B. H. NeumanN
F. WOLF
K. YosHIDA

\section{SUPPORTING INSTITUTIONS}

UNIVERSITY OF BRITISH COLUMBIA

CALIFORNIA INSTITUTE OF TECHNOLOGY

UNIVERSITY OF CALIFORNIA

MONTANA STATE UNIVERSITY

UNIVERSITY OF NEVADA

NEW MEXICO STATE UNIVERSITY

OREGON STATE UNIVERSITY

UNIVERSITY OF OREGON

OSAKA UNIVERSITY

UNIVERSITY OF SOUTHERN CALIFORNIA
STANFORD UNIVERSITY

UNIVERSITY OF TOKYO

UNIVERSITY OF UTAH

WASHINGTON STATE UNIVERSITY

UNIVERSITY OF WASHINGTON

AMERICAN MATHEMATICAL SOCIETY CHEVRON RESEARCH CORPORATION TRW SYSTEMS

NAVAL WEAPONS CENTER 


\section{Pacific Journal of Mathematics}

\section{Vol. 35, No. $1 \quad$ September, 1970}

B. D. Arendt and C. J. Stuth, On the structure of commutative periodic semigroups ..................................... 1

B. D. Arendt and C. J. Stuth, On partial homomorphisms of semigroups .... 7

Leonard Asimow, Extensions of continuous affine functions ............ 11

Claude Elias Billigheimer, Regular boundary problems for a five-term recurrence relation ................................... 23

Edwin Ogilvie Buchman and F. A. Valentine, A characterization of the

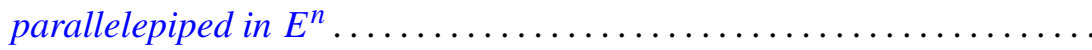
53

Victor P. Camillo, A note on commutative injective rings ............ 59

Larry Jean Cummings, Decomposable symmetric tensors ............. 65

J. E. H. Elliott, On matrices with a restricted number of diagonal values ... 79

Garth Ian Gaudry, Bad behavior and inclusion results for multipliers of type

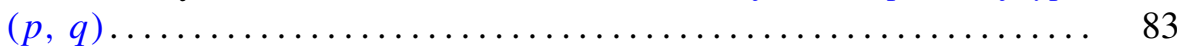

Frances F. Gulick, Derivations and actions .................. 95

Langdon Frank Harris, On subgroups of prime power index . . . . . . . . 117

Jutta Hausen, The hypo residuum of the automorphism group of an abelian

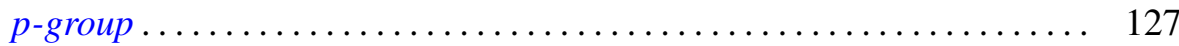

R. Hrycay, Noncontinuous multifuctions . ................... 141

A. Jeanne LaDuke, On a certain generalization of $p$ spaces ............ 155

Marion-Josephine Lim, Rank preservers of skew-symmetric matrices...... 169

John Hathway Lindsey, II, On a six dimensional projective representation of the Hall-Janko group ................................... 175

Roger McCann, Transversally perturbed planar dynamical systems . . . . . 187

Theodore Windle Palmer, Real $C^{*}$-algebras ................... 195

Don David Porter, Symplectic bordism, Stiefel-Whitney numbers, and a

Novikov resolution ....................................... 205

Tilak Raj Prabhakar, On a set of polynomials suggested by Laguerre

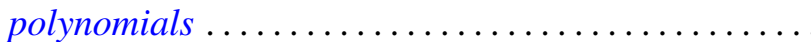

B. L. S. Prakasa Rao, Infinitely divisible characteristic functionals on locally convex topological vector spaces...................

John Robert Reay, Caratheodory theorems in convex product structures ...

Allan M. Sinclair, Eigenvalues in the boundary of the numerical range. .

David R. Stone, Torsion-free and divisible modules over matrix rings ....

William Jennings Wickless, A characterization of the nil radical of a

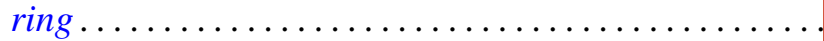

